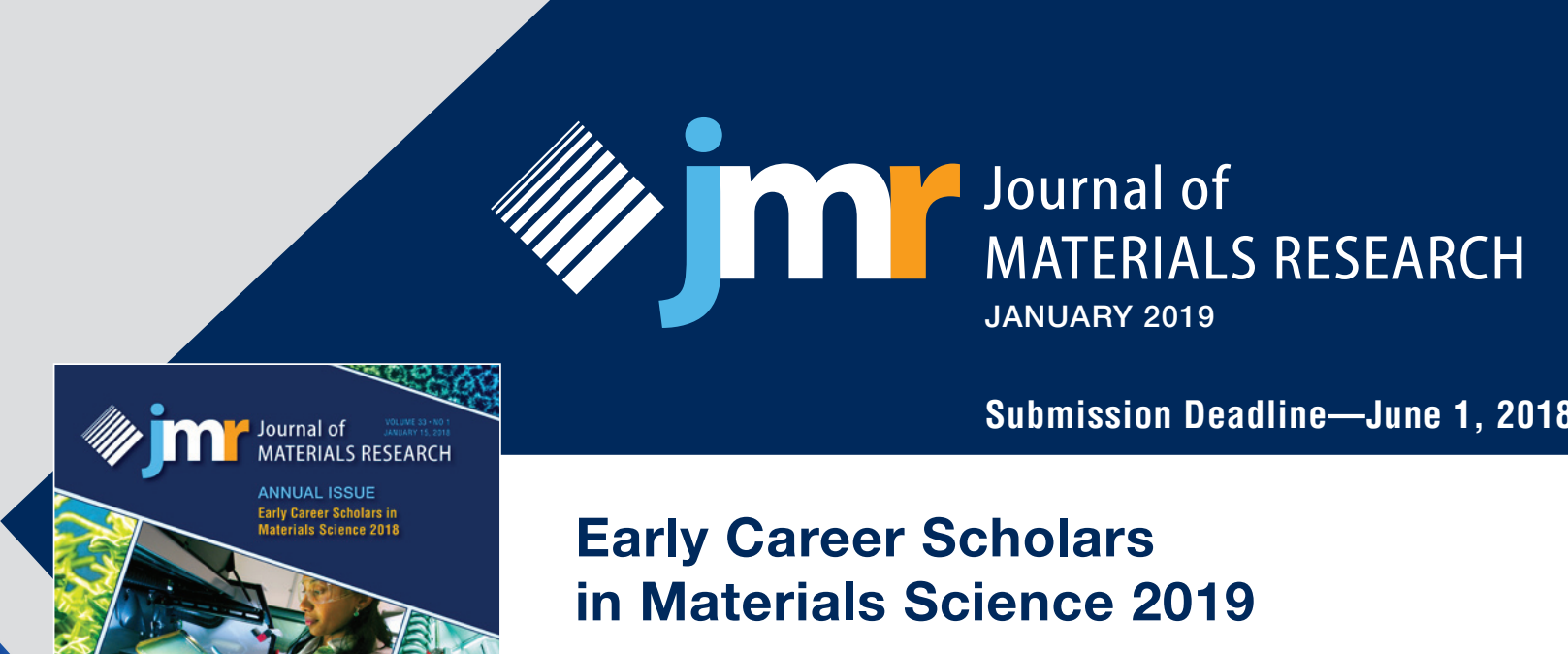

The Fourth Annual JMR Issue to promote outstanding research by future leaders in materials science

This fourth Annual Issue invites full length research and review articles by materials researchers, who have completed their PhD degree but not yet achieved full professorship at the time of submission, for peer review and publication in the January 2019 issue. PhD students are not eligible to submit. The Annual Issue provides a unique opportunity to be highlighted and promoted early in one's research career. To increase attention to these papers, this issue will be published on an open access basis. Although some papers may have multiple authors, only the Early Career Scholar submitting the paper will be identified with a photo and brief bio when the paper is published. Authors from around the world are invited to submit papers that span the topical coverage of $J M R$ including advanced ceramics, metals, polymers, composites, and combinations thereof related to energy, electrical, magnetic, optical, and structural properties and related applications and reporting on:

- Advanced characterization methods and techniques

- Computational materials science when coupled with experimentation

- Fundamental materials science

- Interfacial science as relates to material process understanding and improvements

- Material property enhancements through advances in materials processing

- Material property enhancements through material design (especially Materials Genome related)

- Material combinations and design that improve system performance

- Nanoscience and nanotechnology

\title{
LEAD EDITORS
}

Gary L. Messing, The Pennsylvania State University, USA

Susmita Bose, Washington State University, USA

Jürgen Eckert, Montanuniversität Leoben, Austria

Linda S. Schadler, Rensselaer Polytechnic Institute, USA

\section{MANUSCRIPT SUBMISSION}

To be considered for the issue, the Early Career Scholar must not yet be a full professor at the time of submission. Also, the manuscript must report new and previously unpublished results. Review articles are invited but must be approved by the issue editors before submission (see www.mrs.org/jmr-manuscript-types/ regarding review articles). Manuscripts must be submitted via the JMR electronic submission system by June 1, 2018. Manuscripts submitted after this deadline will not be considered for the issue due to time constraints on the review process. Submission instructions can be found at www.mrs.org/jmr-instructions. Please select "ANNUAL ISSUE: Early Career Scholars in Materials Science 2019" as the manuscript type. Note our manuscript submission minimum length of 3250 words, with at least 6 and no more than $\mathbf{1 0}$ figures and tables. (Additional figures and tables may be submitted as supplemental material.) All manuscripts will be reviewed in a normal but expedited fashion. Papers submitted by the deadline and subsequently accepted will be published in the Special Issue. Other manuscripts that are acceptable but cannot be included in the issue will be scheduled for publication in a subsequent issue of $J M R$.

Papers must be accompanied by a photo (uploaded separately as a high resolution TIF or EPS file) and 200-300 word bio of the Early Career Scholar only. These materials must be submitted along with the original submission of the paper.

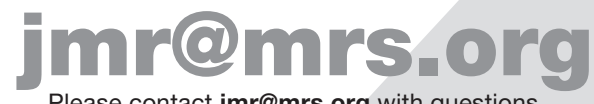

Please contact jmr@mrs.org with questions. 


\section{catalog:americanelements.com}

THE ADVANCED MATERIALS MANUFACTURER \&

\begin{tabular}{|c|c|c|c|c|c|c|c|c|c|c|c|c|c|c|c|c|c|}
\hline 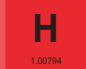 & & & & & & & \multicolumn{5}{|c|}{ 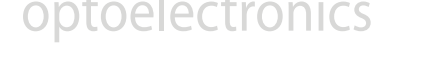 } & \multicolumn{5}{|c|}{ 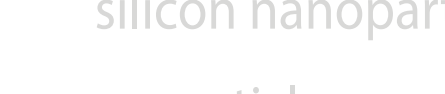 } & \multirow{2}{*}{ 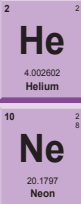 } \\
\hline \multirow{2}{*}{ 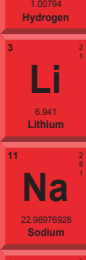 } & \multirow{2}{*}{ 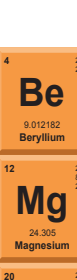 } & \multirow{2}{*}{\multicolumn{10}{|c|}{ surface functionalized nanoparticles }} & B & 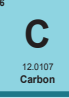 & $\mathbf{N}$ & 0 & 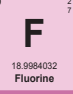 & \\
\hline & & & & & & & & & & & & 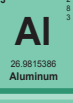 & Si & 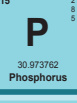 & 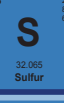 & $\underset{\substack{\text { suses } \\
\text { chereme }}}{\mathrm{Cl}}$ & $\underset{\substack{\text { And } \\
\text { Anom }}}{\mathbf{A r}}$ \\
\hline K & 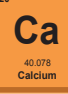 & Sc & 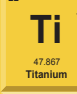 & $\mathbf{v}$ & 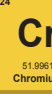 & & $\underset{\substack{\text { ses } \\
\text { tome }}}{\mathrm{Fe}}$ & Co & $\underset{\substack{\text { sind } \\
\text { sinest }}}{\mathrm{Ni}}$ & u & 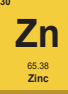 & $\underset{\substack{G a \\
\text { and } \\
\text { catumas }}}{G a}$ & 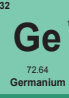 & As & $\mathrm{Se}$ & $\mathrm{Br}$ & 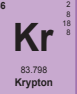 \\
\hline $\mathrm{Rb}$ & 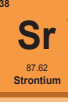 & 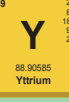 & $" \mathrm{Zr}$ & 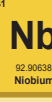 & & & 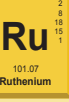 & 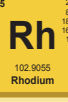 & “ed & Ag & $\mathrm{Cd}$ & $\ln _{\substack{n=0 \\
n=0}}$ & Sn & Sb & $\mathrm{Te}$ & 1 & 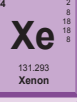 \\
\hline Cs & $\mathrm{Ba}$ &  & 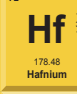 & 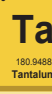 & W & & 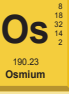 & Ir & 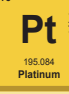 & ${ }^{\mathrm{Au}}$ & $\underset{\substack{i m \in \\
\text { themer }}}{\mathrm{Hg}}$ & 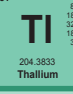 & $\underset{\substack{\text { sho } \\
\text { thed }}}{\mathrm{Pb}}$ & 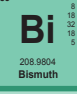 & 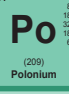 & At & 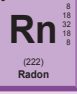 \\
\hline $\mathrm{Fr}$ & 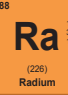 & 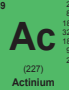 & 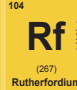 & $\mathrm{Db}$ & 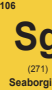 & & 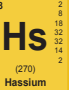 & 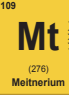 & 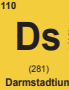 & $\mathbf{R g}$ & 筮 & Uut & 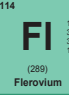 & Uup & 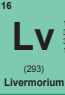 & Uus & Uuo \\
\hline
\end{tabular}

thin film

\begin{tabular}{|c|c|c|c|c|c|c|c|c|c|c|c|c|c|}
\hline $\mathrm{Ce}$ & $\mathrm{Pr}$ & 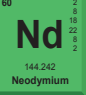 & 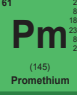 & 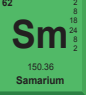 & 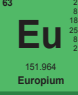 & 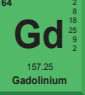 & Tb & Dy & Ho & 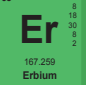 & 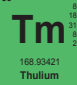 & 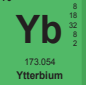 & $\underset{\substack{\text { Lutues } \\
\text { tutume }}}{ }$ \\
\hline 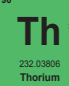 & 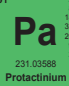 & $\mathbf{U}$ & 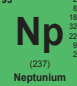 & 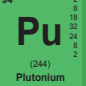 & 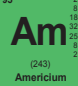 & 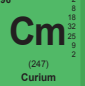 & 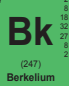 & $\underset{\text { Cf }}{\mathrm{Cf}}$ & 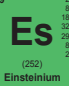 & $\mathrm{Fm}^{\mathrm{Fm}}$ & $\mathrm{Md}$ & 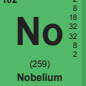 & unevere \\
\hline
\end{tabular}

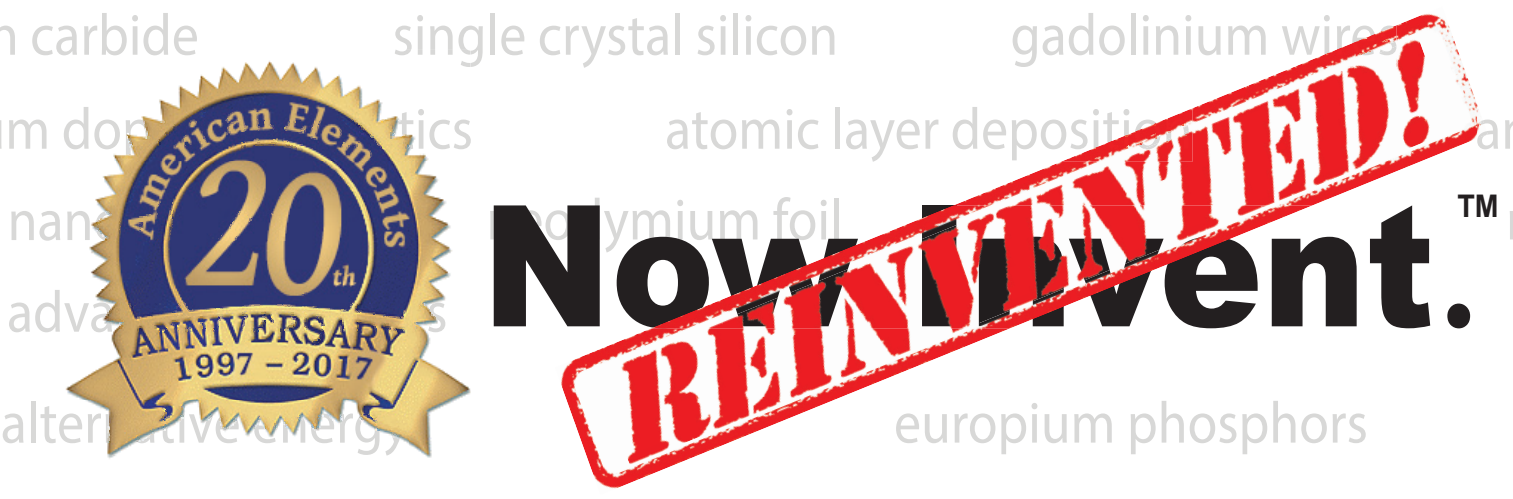

platinum ink

\section{Experience the Next Generation of Material Science Catalogs}

As one of the world's first and largest manufacturers and distributors of nanoparticles \& nanotubes, American Elements' re-launch of its 20 year old Catalog is worth noting. In it you will find essentially every nanoscale metal \& chemical that nature and current technology allow. In fact quite a few materials have no known application and have yet to be fully explored. 